Proc. Indian Acad. Sci., Vol. 86 B, No. 2, August 1977, pp. 139-142, Printed in India.

\title{
Neurochemical correlates of aestivation: Changes in ganglionic protein metabolism in Indian apple snail Pila globosa
}

\author{
INDIRA SINGH and NAYEEMUNNISA \\ Department of Zoology, Bangalore University, Bangalore 560001
}

MS received 27 September 1976; revised 9 March 1977

\begin{abstract}
Changes in RNA, proteins, total free amino acid, ammonia and urea levels were measured in the cerebral, pleuropedal and visceral ganglia of normally active and nine months aestivated snails. RNA, proteins and total free amino acid levels increased on aestivation. Ammonia level showed a decrease on aestivation whereas the level of urea increased in the pleuropedal and visceral ganglia. However, a decrease in urea contents of cerebral ganglion was recorded on aestivation.
\end{abstract}

Keywords. Aestivation; RNA; protein; total free amino acid; Pila globosa.

\section{Introduction}

Considerable information is available on abnormalities of protein metabolism in various tissues of Pila globosa during aestivation (Reddi and Swami 1963; Brahmanandam 1972; Nayeemunnisa 1972; Murali Mohan 1973; Ramanna Rao et al 1974). It has been reported that in aestivating snails protein content and amino acid pool increased in foot, hepatopancreas and mantle (Ramanna Rao et al 1974). Murali Mohan (1973) found manyfold increase in glutamic acid level and a significant decrease in amino transferases in the nervous system of Pila globosa during aestivation. However, no information is available on the ganglionic distribution of protein concentration and changes occurring in their levels during aestivation in Pila globosa. Information available even on other aspects of protein metabolism in aestivated snails is meagre and therefore the present investigation was initiated. The paper protein, total free amino provides information pertaining to the changes occurring in the levels of RNA, acid:, ammonia and urea in the cerebral, pleuropedal and visceral ganglionic pools to Pila globosa during aestivation.

\section{Materials and methods}

Pila globosa were collected from local fresh water ponds and were maintained in glass aquaria at $24 \pm 2{ }^{\circ} \mathrm{C}$ for a week. They were fed on Hydrilla plants. Actively feeding snails were aestivated by burying them in dry sand for nine montbs.

Changes occurring in the levels of RNA, protein, total free amino acids, ammonia and urea were studied in the cerebral, pleuropedal and visceral ganglia of normally active and aestivated (experimental) snails. 
The animal was dissected on a wax plate kept on ice blocks, cerebral, pleuropedal and visceral ganglia were isolated and kept in cavity glasses at $0^{\circ} \mathrm{C}$. The ganglia were pooled from five animals and weighed in ice cold Pila Ringer (Lal and Agarwal 1969) and immediately used for analyses.

RNA contents were extracted by the method of and estimated by orcinol colour reaction following the colorimeteric procedure described by Glick (1964).

Proteins were precipitated by TCA and estimated following the method of Oser (1965).

Quantitative analysis of total free amino acids was carried out with the deproteinized sample using the method of Danielson as given by Oser (1965).

The levels of ammonia and urea were determined by Vansylke and Cullen's aeration method as described by Oser (1965).

\section{Results}

In general, the visceral ganglion has the highest RNA level in active and also in aestivated snails than the other ganglia. The RNA content is comparatively low in the pleuropedal ganglia of both normally active and aestivated snails (table 1).

On aestivation a marked increase was observed in the RNA level of all the ganglia studies. However, the magnitude of response was the highest in the cerebral ganglion, and the pleuropedal ganglion exhibited the least response on a comparative basis (table 1 ).

As a consequence of aestivation, the protein content increased in the cerebral, pleuropedal and viscereal ganglia. However, the increase in the cerebral ganglion was the maximum $(+18)$ and the least increase $(+13)$ was found in the pleuropedal ganglion.

Total free amino acid level in the cerebral ganglia of both the active and aestivated snails are higher compared to the pleuropedal and visceral ganglia. The visceral ganglion exhibited the lowest level of free amino acids (table 1).

A marked increase in total free amino acid level was exhibited on aestivation. Maximum increase was observed in the pleuropedal ganglion $(+102)$ while the least response $(+85)$ was shown by the cerebral ganglion (table 1$)$.

Ammonia and urea levels were the highest in the pleuropedal ganglia of both the control and experimental snails, and lowest in the visceral ganglion of control animal. The cerebral ganglion exhibited maximum ammonia levels in the experimental (aestivated) snails. However, urea levels were comparatively low in the cerebral ganglion of aestivated snails (table 1).

An elevation in the urea level of pleuropedal and visceral ganglia was observed during aestivation whereas the cerebral ganglion showed a depletion in its urea level (table 1).

\section{Discussion}

There was an increase in RNA, proteins and total free amino acid levels, while the levels of ammonia decreased on aestivation.

Earlier studies on aestivation indicated that the dehydration or prolonged osmotic stimulus leads to a general activation of RNA and protein synthesizing machinery 
Ganglionic protein metabolism in Pila Globosa

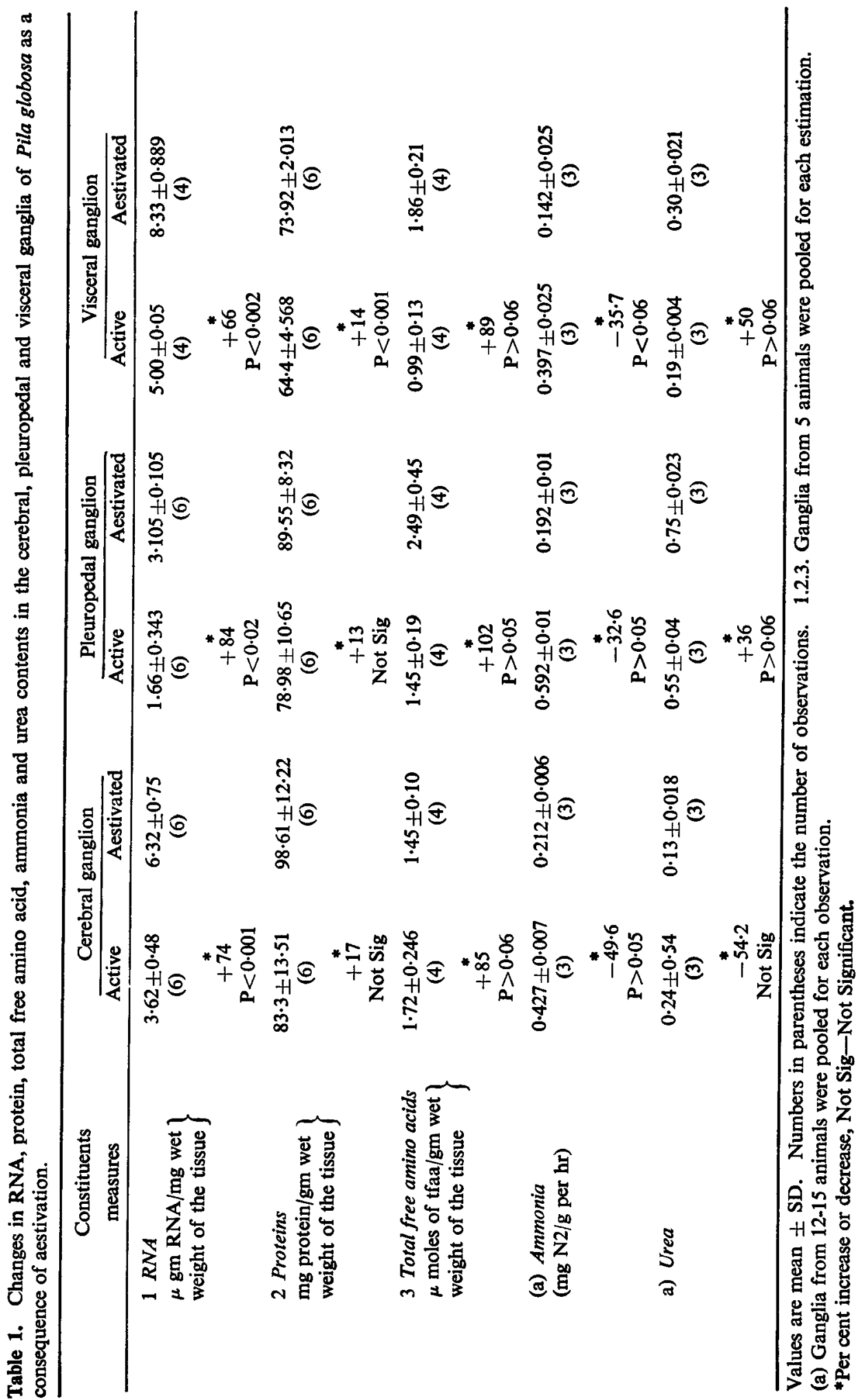


(Brahmanandam 1972; Sacho 1970). In the present investigation, aestivation is known to effect osmotic properties and leading to dehydration in Pila globosa (Reddi and Swami 1963; Brahmanandam 1972; Nayeemunnisa 1972, 1975; Murali Mohan 1973; Ramanna Rao et al 1974).

An interesting feature is the increase in the free amino acids of cerebral, plueropedal and visceral ganglia of aestivated snails. Murali Mohan (1973) had found a similar increase in the free amino acid content of the nervous system of Pila globosa during aestivation. This may be correlated to the osmatic properties of the snail during aestivation. In Pila globosa there is marked dehydration during aestivation (Reddi and Swami 1963; Nayeemunnisa 1972; Murali Mohan 1973; Meenakshi 1956) which results in general increase of the free amino acid content in tissues during aestivation. The decrease in ammonia level (table 1) may be the direct consequence of the above, to cope up with the non-availability of water for ammonia excretion.

However, contrary to expectation, on elevation in the levels of urea was observed in the pleuropedal and visceral ganglia, while a depletion of urea level was observed in the cerebral ganglion during aestivation. The cause for such an increase and decrease in the urea levels of the ganglia during aestivation is not known. But, it is possible that in the nervous tissue, the major mechanism for the removal of ammonia is the formation of glutamine (Ganong 1969). Therefore, the decrease in the levels of urea observed during aestivation, in the present study points to this possibility.

\section{Acknowledgement}

Authors are thankful to A R Kasturi Bai of the Dept. of Zoology, Bangalore University, Bangalore, for facilities in conducting this study.

\section{References}

Brahmandam V 1972 Some biochemical studies on the gastropod muscle proteins with particular reference to aestivation. Ph.D. dissertation (Bangalore University)

Ganong W F 1969 in Review of Medical Physiology (Tokyo: Maruzen Asian Edition)

Gliok D 1964 Methods of Biochemical Analysis (New York: Interscience Pub.) Vol. 1

Lal M B and Agarwal R A 1969 A saline medium for maintaining the isolated heart of Pila globosa (Swainson); Proc. Indian Acad. Sci. B67 1-9

Meenakshi V R 1956 Physiology of hibernation of the apple-snail Pila Virens (Lamarck); Curr. Sci. 25 321-322

Murali Mohan P 1973 Studies on some physiological and biochemical aspects of aestivation of a selected gastropod with special reference to aestivation; Ph.D. dissertation (Sri Venkateswara Univ.)

Nayeemunnisa 1972 Aspects of nitrogen excretion during aestivation in amphibious snail Pila globosa I. Evidence of neuroendocrine involvement in the pattern of excretion; Comp. Biochem. Physiol. A42 1049-1053

Nayeemunnisa 1975 Aspects of nitrogen excretion during aestivation in amphibious snail Pila globosa II. Evidence for enzyme increase susceptibility to neuroendocrine influence; Indian J. Exptl. Biol. 13 309-311

Oser B 1965 in Hawk's Physiological Chemistry (Tata McGraw Hill Pub. Co.)

Raghupathirama Reddy S and Swami K S 1964 On the occurrence of a heme pigment in the stomach of the amphibious snail Pila Globosa; Curr. Sci. 33 495-496

Ramanna Rao K V, Murali Mohan P and Swami K S 1974 Cardiac activity during aestivation of the snail Pila globosa (Swainson); Curr. Sci. 43 453-454

Sacho H 1970 in Handbook of Neurochemistry ed. A Lajtha (New York, London: Plenum Press) Vol. IV, p. $373-428$ 